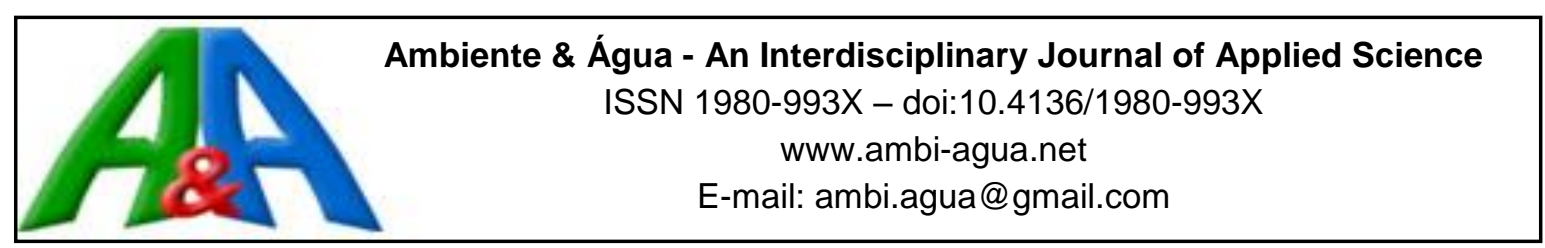

\title{
Sinais de um problema crônico: a governança hídrica carece promover os comitês de bacias, coordenar planos e gerir informações
}

\author{
doi:10.4136/ambi-agua.2044
}

Received: 14 Nov. 2016; Accepted: 14 Oct. 2017

\author{
Clécio Barbosa Souza Júnior ${ }^{1 *}$; Marianna Siegmund-Schultze ${ }^{1}$; \\ Johann Köppel' ${ }^{1}$, Maria do Carmo Sobral ${ }^{2}$ \\ ${ }^{1}$ Berlin Institute of Technology (TU), Berlin, Germany \\ Environmental Assessment and Planning Research Group. E-mail: junior@ campus.tu-berlin.de, \\ m.siegmund-schultze@tu-berlin.de, johann.koeppel@tu-berlin.de \\ ${ }^{2}$ Universidade Federal de Pernambuco (UFPE), Recife, PE, Brasil \\ Departamento de Engenharia Civil.E-mail: msobral@ufpe.br \\ *Autor correspondente
}

\section{RESUMO}

A atual governança Hídrica no Brasil contribui para o aumento dos problemas oriundos da escassez hídrica na bacia do São Francisco, majorando os conflitos pelo uso da água. Este artigo tem o objetivo de avaliar alguns desafios e oportunidades da governança hídrica na bacia do São Francisco com base numa robusta coleta e análise de dados nos mais diversos órgãos e instituições em escalas nacional, estadual e de bacia. A metodologia inclui a análise da interação interinstitucional através do exame da coordenação dos planos de recursos hídricos, a análise da implementação dos comitês de bacias dos rios afluentes e a quantidade e qualidade acessível de dados relativos à gestão de águas. A maioria dos planos dos rios afluentes vigentes foi elaborada pelo poder público e consequentemente, boa parte dos planos analisados apresentam propostas com divergências regionais em suas diferentes escalas de planejamento e apresentam falhas na sinergia entre as ações dos diversos planos. Além disso, a região semiárida padece com a baixa implantação dos comitês de bacias de rios afluentes e a bacia como um todo sofre com a falta de um mecanismo apropriado de produção, atualização e compartilhamento de informações relevantes para a gestão hídrica. Portanto, faz-se necessário aprimorar o mecanismo de articulação entre os planos com o uso de ferramentas de avaliação integrada e de contexto decisório, fortalecer a participação pública através de comitês de rios afluentes e a adoção de uma plataforma de dados colaborativa donde se possa compartilhar informações geradas em diferentes setores da sociedade.

Palavras-chave: disponibilização de informação, integração interinstitucional, promoção da participação pública.

\section{Signs of a chronic problem: need for water governance to promote basin committees, coordinate plans and manage information}

\section{ABSTRACT}

The current water governance in Brazil contributes to the increase of water scarcity problems in the São Francisco basin, contributing to conflicts over water use. This paper assesses the challenges and barriers of water governance in the São Francisco river basin based 
on a robust collection and analysis of data from various repositories and institutions at the national, state, river basin and municipal levels. The methodology includes an analysis of institutional interaction by examining the coordination of water resources plans, a review of the implementation of basin tributary committees and the quantity and quality of available data related to water management. Most tributaries' river plans were developed by the government; therefore, many of the plans analyzed conflicted with other regional plans due to a lack of coordination of proposed actions. In addition, the semiarid region has implemented few tributary river committees and the basin as a whole lacks an appropriate mechanism for generating, updating and sharing comprehensive information relevant to water management. It is therefore imperative to improve coordination between plans using integrated assessment and decision-making tools, and to reinforce public participation through tributary committees. Further, a collaborative data platform should be adopted, from which it will be possible to share information generated in different sectors of society.

Keywords: information availability, interinstitutional integration, promotion of public participation.

\section{INTRODUÇÃO}

Segundo a OECD (2015), a governança da água é um reflexo da cultura, regime político e organizacional de um país. Talvez por essa razão a gestão hídrica ainda seja um negócio inacabado no Brasil, que desde sua descoberta até os anos de 1822 - período colonial - o país foi marcado por intensas atividades exploratórias aos recursos naturais. Segundo OECD (2015), a nova constituição brasileira de 1988 é tida como um marco da governança de água no Brasil, pois inclui um importante capítulo sobre meio ambiente e prevê o Sistema Nacional de Gerenciamento dos Recursos Hídricos (SINGREH). Com a conferência das nações unidas sobre o meio ambiente no Rio 92, criou-se um cenário que possibilitou mais tarde a instituição de uma política de recurso hídrico no país através da Lei 9.433/97. A lei procurou reverter esse paradigma da cultura exploratória voltada para a comercialização e exaustão dos recursos hídricos e providenciou uma plataforma para a implementação do conceito e princípios do Gerenciamento Integrado dos Recursos Hídricos (GIRH). Segundo Veiga e Magrini (2013), o Brasil alcançou notáveis progressos após a instituição desta Lei Federal, porém ainda não se percebe plenamente os benefícios econômicos, sociais e ambientais esperados.

Não existe consenso de escopo, definição e medida da governança hídrica, isso pode ser notado analisando os contrastes existentes entre definições da OECD (2015), Biswas and Tortajada (2010), entre outros. O conceito de governança no setor hídrico é o novo paradigma de gestão de recursos hídricos. Nesheim et al. (2010) citam que uma efetiva governança das águas está relacionada com uma integração intersetorial e com uma coordenação entre as dimensões institucionais de diferentes escalas. A OECD (2015), entre outros aspectos, destaca que a gestão hídrica deve ser tratada em escala adequada; menciona a importância da disponibilidade, efetividade e acessibilidade de dados relativos à gestão das águas; e relata que a promoção do envolvimento das partes interessadas e usuários é um ponto crucial para uma gestão descentralizada e participativa.

No Brasil a feitura da gestão de recursos hídricos ainda é incipiente porque existem incompatibilidades entre fronteiras administrativas e limites hidrológicos causando problemas e inconsistências de ordem legal e administrativas e a OECD (2015) destaca ainda que "os diversos planos de recursos hídricos em nível nacional, estadual, local e de bacias são mal coordenados e não chegam a ser colocados em prática". Além disso, explana a necessidade de fomentar um mecanismo que envolva os atores em tomadas de decisões inclusiva e transparentes e sublinha a lacuna no acesso a informações, na qual carece de desenvolvimento de plataformas de informações confiáveis, consistentes e transparentes para apoiar tomadores de decisão. 
Segundo Silva e Cirilo (2011), a integração interinstitucional numa bacia pode ser analisada pela integração entre os planos de recursos hídricos. Os planos são elementos essenciais à gestão do setor hídrico, uma vez que segundo a Lei 9.433/97, são os planos diretores que visam fundamentar e implementar a PNRH. Moss (2003) e Mitchell (2004) têm analisado a importância da coordenação vertical entre os planos territoriais e de bacias. Eles destacam lacunas, principalmente na base legal que impede uma boa coordenação entre planos, bem como discutem a credibilidade que se é dada aos planos de bacias locais e regionais quando os mesmos são elaborados de forma coordenada e integrada aos planos territoriais. Carter et al. (2004) ressaltam que a gestão integrada entre os diferentes níveis é um grande desafio, principalmente em nível local. E destacam que decisões sobre o uso da terra afetam diretamente os sistemas hidrológicos e essa relação direta não é tacitamente reconhecida. Iribarnegaray e Seghezzo (2012) destacaram que uma boa governança também contempla a disponibilidade e boa qualidade das informações contidas em web sites de instituições relacionadas com a gestão das águas.

A região hidrográfica do rio São Francisco é uma das mais importantes do Brasil e é a mais representativa do Nordeste brasileiro. Tem aproximadamente $639000 \mathrm{~km}^{2}$, que equivale a $8 \%$ do território nacional abrangendo 507 municípios distribuídos em sete Unidades Federativas (Bahia, Minas Gerais, Pernambuco, Alagoas, Sergipe, Goiás e Distrito Federal) (CBHSF, 2016). E é dividida em quatro regiões fisiográficas (Alto, Médio, Submédio e Baixo São Francisco). A bacia apesar de ser uma das poucas regiões hidrográficas que o comitê, a agencia de água, e todos os instrumentos da Política Nacional de Recursos Hídricos foram instituídos, sofre com diversos problemas devido aos conflitos de uso das águas. Os setores energéticos e agrícolas são os principais responsáveis pela maioria dos conflitos. O primeiro por reduções da vazão a jusante dos reservatórios, que vem causando impactos sobre as condições de vida e de trabalho das comunidades ribeirinhas e para o ecossistema; e o segundo apresenta um planejamento pouco coordenado com outros usuários e às vezes um uso de água pouco eficiente.

Em escala local, dos 29 planos diretores de rios afluentes existentes na região São Francisco, 20 foram elaborados entre os anos de 1993 e 1998 e até momento não foram atualizados, pois conforme as suas validades ainda não requerem atualização. Neves e Cordeiro Netto (2005) analisaram a eficiência de 20 planos de recursos hídricos no Brasil - sendo quatro deles na região da bacia do rio São Francisco - e concluíram que os mesmos têm se mostrado com baixa eficiência de implementação. Além disso, Silva e Cirilo (2011) perceberam uma frágil integração entre o plano de recursos hídricos do rio São Francisco e de seus planos de rios afluentes, identificando um relevante número de ações presentes no Plano de Bacia Hidrográfica do São Francisco (PBHSF 2004-2013) e que estão ausentes nos Planos Diretores de Bacias Hidrográficas (PDBHs) dos rios afluentes. Outro aspecto importante, em escala da bacia do São Francisco, é a lacuna na implantação dos comitês de bacias, especialmente na região semiárida, dificultando a participação dos interessados nos processos de negociação e de tomada de decisão. Siegmund-Schultze et al. (2015) destacam ainda que apesar do Comitê de Bacia Hidrográfica do São Francisco (CBHSF) ser ativo, a participação não é homogênea, ou seja, não representa de forma equitativa todas as regiões da bacia.

$\mathrm{O}$ artigo pretende construir uma abordagem quantitativa para avaliar a eficácia da governança hídrica, diferenciada por região, a fim de detectar principais deficiências e recomendar mudanças. Três aspectos foram selecionados para testar a abordagem: a coordenação vertical entre planos de bacia, o compartilhamento de informações e a implementação de comitês de bacia. A hipótese defende que esses três aspectos analisados são representativos a ponto de se identificar, ao menos, as disparidades na gestão entre as diferentes regiões fisiográficas e níveis administrativos dentro da região do rio São Francisco. 


\section{MATERIAIS E MÉTODOS}

Considerando uma abordagem metodológica que destaca três importantes aspectos para mensurar a capacidade dos estados e das bacias de rios afluentes em gerenciar os recursos hídricos na bacia do rio São Francisco, esse artigo baseia-se em pesquisas bibliográficas e documentais para a) analisar a integração interinstitucional através do exame da articulação entre planos de recursos hídricos; b) avaliar sob o ponto de vista quantitativo e qualitativo as informações contidas nos web sites de cinco das mais importantes instituições relacionadas com a gestão das águas na bacia, e; c) analisar a implementação dos comitês de bacias hidrográficas dos rios afluentes ao rio São Francisco.

\subsection{Analise da Integração Interinstitucional}

$\mathrm{Na}$ análise da integração interinstitucional, foi avaliado a articulação entre os planos estaduais e de bacias com base em metodologia proposta por Lanna et al. (2002) que propõe duas formas: a análise por agregação e por coordenação de planos. A primeira enfatiza que o plano nacional e os planos estaduais sejam uma agregação dos planos diretores de bacias de rios interestaduais e de rios afluentes, porém o próprio autor argumenta que a agregação dos planos não deve ser entendida como uma mera repetição ou agrupamento de outros, e sim que os primeiros planos - o Plano Nacional, Interestaduais e Estaduais - tenham ênfase na organização das atividades, na articulação das demandas e na coerência das estruturas de planejamento e gestão. E devem ser elaborados com menos detalhes que os planos diretores de bacias dos rios afluentes que atuam em âmbito mais restrito. Dessa forma, evita-se que as demandas operacionais que devem ser solucionadas pelos planos de bacias sejam transferidas ou assumidas pelas esferas dos planos estaduais e nacional, levando em conta a importância na divisão de responsabilidade entre as diversas esferas administrativa na bacia (ANA, 2013). Segundo Silva e Cirilo (2011), a análise dos planos por agregação gera um conflito decisório entre os órgãos envolvidos e que parte do pressuposto de que não há conflitos de demandas e interesses nas bacias envolvidas. Portanto, a segunda forma de análise, a análise da integração por coordenação, é a mais apropriada para o referente artigo, pois consiste num processo no qual "as demandas dos âmbitos mais restritos são processados nos âmbitos mais amplos, gerando orientações, na forma de diretrizes de planejamento, que deverão ser acatadas" (Lanna at al, 2002; p.13). Nesse contexto, Silva e Cirilo (2011) apresentam resultados contundentes na avaliação da integração entre os planos nacional, estaduais e da bacia do rio São Francisco.

Para o uso da referida metodologia, o artigo pressupõe que os planos estaduais são elaborados com base em reivindicações de seus comitês de bacias de rios afluentes e, portanto, não avalia se as diretrizes dadas pelos planos estaduais são representativas para as particularidades de cada bacia. E é fundamentada no princípio de que os órgãos estaduais de recursos hídricos (Institutos, Companhias, Agências de Água, Secretarias de Recursos Hídricos, entre outros) processam as demandas das bacias de rios estaduais e elaboram os seus planos estaduais de recursos hídricos (PERHs), e os Conselhos Estaduais de Recursos Hídricos (CERHs) supervisionam e aprovam o referido plano, conforme determina a política nacional. A Figura 1 apresenta as premissas e questionamentos adotados no processo de análise da integração por coordenação entre os planos. 


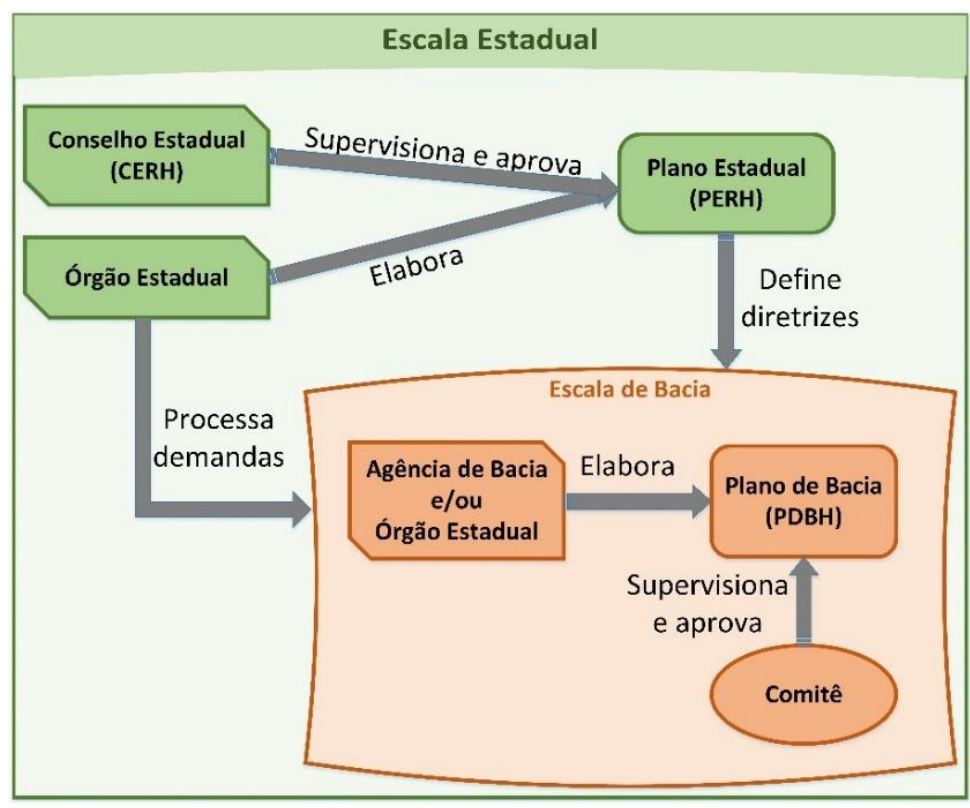

Figura 1. Articulação entre planos de recursos hídricos em escala estadual.

O critério para seleção dos planos de bacias analisados foi baseado no ano de elaboração e atualização dos mesmos. Todos os planos diretores elaborados antes dos seus respectivos planos estaduais foram excluídos da análise, a fim de validar as premissas e metodologia expostas anteriormente, donde planos locais são elaborados com base nas diretrizes definidas pelos planos mais abrangentes. Outro importante critério levou em consideração o ano em que foi estabelecida a Lei 9.433, ou seja, apenas os planos de bacias elaborados ou atualizados após 1997 foram escolhidos para a análise, pressupondo-se que os mesmos foram elaborados com base no novo modelo da política de recursos hídricos.

Após a seleção dos planos estaduais e seus respectivos planos de bacias foram identificados todos os programas e subprogramas existentes em ambos os planos, a fim de conhecer seus objetivos, diretrizes e ações propostas. Em seguida, avaliou-se se os programas/subprogramas definidos pelos planos estaduais (PERHs) são iguais ou semelhantes ao seu respectivo plano de bacia de rios afluentes, ou se estão ausentes. E foi utilizada uma simbologia para classificá-los. Os programas/subprogramas são considerados iguais (PI) quando se constatam mesmos objetivos em ambos e o plano estadual promova diretrizes que apoie as ações de seu respectivo plano de bacia. Os programas e subprogramas semelhantes (PS) guardam similaridades em seus objetivos, porém não fica claro se o plano local utiliza as diretivas de seu plano mais abrangente ou se o plano mais amplo fornece diretrizes ao mais restrito. E os ausentes (PA) não possui nenhuma correlação entre o seu plano estadual e respectivo plano de bacia. Para finalizar a análise da integração entre os planos, foi utilizada uma escala percentual de 0 a $100 \%$ para determinar o grau de coordenação entre os planos. Silva e Cirilo (2011), que investigaram a integração entre o plano de bacia do São Francisco e os planos estaduais da Bahia, Pernambuco e Sergipe fundamentaram que uma boa integração entre os planos poderiam ser medidos pela porcentagem dos programas e subprogramas iguais (PI) entre o plano de maior abrangência e o de menor escala. Portanto, o presente artigo usa a escala em que considera a porcentagem dos programais iguais (PI) entre os PERHs e seus respectivos planos de bacia de rios afluentes para definir se a integração por coordenação entre os planos são inaceitáveis, insuficiente, boa ou excelente. Conforme a Tabela 1. 
Tabela 1. Integração entre os PERHs e seus respectivos planos de bacia de rios afluentes.

\begin{tabular}{l|cc}
\hline & Iguais $(\mathbf{P I})$ & Integração por coordenação \\
\cline { 2 - 3 } Programas / & $\mathbf{P I} \leq 25 \%$ & Inaceitável \\
Subprogramas & $25 \%<\mathbf{P I} \leq 50 \%$ & Insuficiente \\
& $50 \%<\mathbf{P I} \leq 75 \%$ & Boa \\
& $\mathbf{P I}>75 \%$ & Excelente \\
\hline
\end{tabular}

\subsection{Avaliação da Implementação dos Comitês}

O segundo aspecto, analisa a oportunidade de participação dos usuários e da sociedade civil nas decisões de gestão da bacia. Esta variável avalia o número de comitês de rios afluentes implementados, considerando o potencial que os comitês têm na descentralização da decisão e no compartilhamento de interesses, que são relevantes pontos no avanço da governança hídrica de uma bacia. Destacando-se que a bacia do São Francisco é extensa e possui características e conflitos bastante diversos, os comitês de bacias de rios afluentes são de grande valia, visto que trabalham em menor escala e podem discutir com maior propriedade os problemas de dimensão local. A escala de análise varia de 0 a $100 \%$, e considera o número de comitês implementados em relação às unidades de planejamento hídricos existentes na bacia.

\subsection{Exame das Informações Disponibilizadas nos Web sites}

A comunicação adequada entre instituições e setores usuários é um ponto crucial na gestão dos riscos e conflitos. Portanto, esse terceiro aspecto trata da avaliação sob o ponto de vista quantitativo e qualitativo das informações contidas nos web sites (WS) de cinco das mais importantes instituições relacionadas com a gestão das águas na bacia. A OECD (2015) destaca a importância em produzir, atualizar e compartilhar dados e informações oportunas, coerentes, e politicamente relevantes e relacionados com a água. Com isso, a disponibilidade, efetividade e acessibilidade dos dados disponíveis nos web sites para os atores da bacia foram analisadas através da avaliação de 10 critérios técnicos apresentados por Codina (2000) e adaptados com algumas considerações da OECD (2015) e Iribarnegaray e Seghezzo (2012). Esses critérios abordam os seguintes pontos:

1- Rigor: Refere-se ao cuidado com que a informação tenha sido preparada. Ou seja, analisa a ausência de erros ortográficos e conceituais, menções de datas e autores e apresentação de estatísticas de acesso;

2- Edição: Este indicador refere-se ao grau mais ou menos evidente em que a informação tenha sido processada, revisada, antes de ser publicada. Se a informação teve tratamento editorial, se o recurso foi identificado e datado;

3- Interesse intrínseco: Checa se o site tem informações de interesse intrínseco ou o interesse é trivial. Foi avaliado se possui conteúdos importantes e se desperta o interesse do público alvo;

4- Volume: Verifica se a informação tem um nível adequado de cobertura da informação, tendo em conta o assunto ou domínio coberto.

5- Exaustividade: Este indicador refere-se ao grau em que a informação é apresentada. Ou seja, verifica a disponibilidade de dados de operação (hidrogeológicos, meteorológicos e hidrológicos); dados sobre os direitos de uso, licenças, outorgas; disponibilização de planos de bacias; dados administrativos (registros relacionados com pessoal, equipamento e finanças); aspectos técnicos e de gestão (resultados de pesquisas e estudos de campo, etc.); disponibilização de materiais de promoção para aumentar a conscientização, melhorar a motivação e mudança de comportamento, entre outros;

6- Atualização: Indica a frequência e a data da última atualização; 
7- Sistematização: Analisa se as informações são descritas com a mesma estrutura básica e com padrões de publicação e bancos de dados. E se o site possui uma plataforma de dados que permita que diferentes instituições incluam dados de forma colaborativa e padronizada;

8- Originalidade: Analisa se os recursos, tais como mapas, gráficos, infográficos, são originais ou exclusivos para o site;

9- Responsabilidade: Verifica se a informação contém indicações claras e explícitas de autoria e/ou responsabilidade do recurso intelectual;

10- Importância: Procura sinais de que a web site é criada, produzida, realizada, etc., por um órgão ou instituição de renome em seu campo.

Codina (2000) classifica cada critério de acordo com sua importância criando três níveis. Critérios de nível um são critérios mínimos e necessários, de nível dois são critérios notáveis e de nível três são critérios excelentes. E numa abordagem similar à de Iribarnegaray e Seghezzo (2012), a análise do acesso a informação na bacia é estudada através de um score que avalia a qualidade, quantidade e autoria dos dados de cada site selecionado. Considerando pesos um, dois e três para os critérios de níveis um, dois e três respectivamente, e através de uma média ponderada simples foi classificado cada site numa escala que varia de 0 a $100 \%$. Essa escala de classificação determina se os dados disponíveis são: inaceitável, insuficiente, bom ou excelente, conforme a Tabela 2 .

Tabela 2. Informações disponíveis nos web sites.

\begin{tabular}{cc}
\hline Informações dos Web sites & Quantidade e qualidade dos dados \\
\hline $\mathbf{W S} \leq 25 \%$ & Inaceitável \\
$25 \%<\mathbf{W S} \leq 50 \%$ & Insuficiente \\
$50 \%<\mathbf{W S} \leq 75 \%$ & Boa \\
$\mathbf{W S}>75 \%$ & Excelente \\
\hline
\end{tabular}

Em resumo, a Tabela 3 apresenta os três aspectos da governança considerados no presente artigo, bem como seus indicadores, variáveis e dados necessários para análise.

Tabela 3. Aspectos analisados na governança hídrica.

\begin{tabular}{llll}
\hline $\begin{array}{l}\text { Aspectos da } \\
\text { Governança }\end{array}$ & Indicadores & Variáveis & Dados necessários \\
\hline $\begin{array}{l}\text { Interação } \\
\text { interinstitucional }\end{array}$ & $\begin{array}{l}\text { Integração por } \\
\text { coordenação entre } \\
\text { planos de bacias }\end{array}$ & $\begin{array}{l}\text { Programas iguais }(\mathbf{P I}), \\
\text { semelhantes (PS) ou ausentes } \\
(\mathbf{P A}) \text { entre planos de diferentes } \\
\text { escalas }(0-100 \%)\end{array}$ & $\begin{array}{l}\text { Planos de bacias } \\
\text { estaduais e de rios } \\
\text { afluentes }\end{array}$ \\
$\begin{array}{l}\text { Participação } \\
\text { pública }\end{array}$ & $\begin{array}{l}\text { Implementação de } \\
\text { comitês }\end{array}$ & $\begin{array}{l}\text { Implementação de comitês dos } \\
\text { rios afluentes }(0-100 \%)\end{array}$ & $\begin{array}{l}\text { Ata de fundação do } \\
\text { comitê }\end{array}$ \\
$\begin{array}{l}\text { Acesso à } \\
\text { informação }\end{array}$ & Web site & $\begin{array}{l}\text { Qualidade e quantidade de } \\
\text { dados nos sites institucionais } \\
(0-100 \%)\end{array}$ & $\begin{array}{l}\text { Informações técnicas } \\
\text { obtidas dos web sites }\end{array}$ \\
\hline
\end{tabular}

\section{RESULTADOS E DISCUSSÃO}

\subsection{Integração por Coordenação entre Planos de Bacias}

Em uma consulta aos órgãos estaduais de recursos hídricos e à ANA, contabiliza-se atualmente um universo de 34 planos de recursos hídricos elaborados na bacia hidrográfica do rio São Francisco em diferentes níveis administrativos. E diversos outros em fase de licitação, 
contratação ou elaboração. Nota-se que dez planos de bacias foram elaborados antes ou no ano da promulgação da Lei 9.433/97, sendo a grande maioria do estado da Bahia; e dez planos estão com o horizonte de planejamento vencido (pondera-se que alguns deles estão em processo de elaboração e licitação). O plano nacional (PNRH) é o plano de recursos hídricos que atua em escala nacional e estabelece em linhas gerais quatro componentes, 13 programas subdivididos em 30 subprogramas e tem como área de atuação as 12 regiões hidrográficas brasileiras definidas pelo CNRH. A integração entre as diretrizes do PNRH e do plano da bacia hidrográfica do rio São Francisco (PBHSF 2004-2013) foram analisadas por Silva e Cirilo (2011), adotando-se os programas e ações entre ambos como elementos de comparação. O PNRH foi tomado como referência por se tratar de um plano mais amplo e o critério utilizado na análise da integração foi a presença ou ausência de mesmo programa em ambos os planos e a presença de programas similares. Considerando que alguns programas foram excluídos das análises por se tratar de programas específicos a áreas com características particulares, os autores concluíram que o PBHSF 2004-2013 possui um bom nível de coordenação os programas e diretrizes nacionais. Incluindo programas similares e idênticos, $80 \%$ das ações guardavam similaridades. Com critérios de análise similar, os mesmos autores também apresentam resultados da análise entre PBHSF 2004-2013 e os planos estaduais (PERHs) dos estados da Bahia, Pernambuco e Sergipe, localizados respectivamente nas regiões fisiográficas do Médio, Submédio e Baixo São Francisco. A Bahia foi o estado que apresentou uma melhor coordenação entre o seu plano estadual e o PBHSF, com 50\% das atividades iguais em ambos os planos. Vale enfatizar que a atualização do Plano de Bacia Hidrográfica do São Francisco foi recentemente aprovado em setembro de 2016 (PBHSF 2016-2025) e que uma nova análise seria necessário para avaliar o nível de integração entres o PNRH e os PERHs. O presente artigo complementa essa análise, fazendo uma análise em escala estadual, entre os Planos Estaduais e seus respectivos Planos de rios afluentes. Aplicando os critérios pré-estabelecidos anteriormente, foram cabidos 10 planos diretores de bacias hidrográficas para análise, seis do estado mineiro, três do estado pernambucano e um alagoano.

O PERH de Minas Gerais foi aprovado em 2010 e é dividido em quatro componentes, 13 programas e 29 subprogramas. A preocupação com a interação dialética e convergências entre os planos em diferentes escalas é um tópico tratado dentro do plano estadual. Considerando o mesmo critério utilizado por Silva e Cirilo (2011), 15 subprogramas foram excluídos da análise por se tratarem de ações específicas de âmbito estadual. E, portanto, foram identificados 14 subprogramas nos quais poderiam subsidiar e respaldar as ações dos planos diretores das bacias selecionadas. Os resultados mostram que os planos dos rios Urucuias, Pandeiros e das Velhas apresentaram uma boa articulação como o plano estadual mineiro, com destaque para o plano do rio das Velhas que mais de $90 \%$ de suas diretrizes foram baseadas nos programas do plano estadual, conforme explicita a Tabela 4.

No estado de Pernambuco, os planos de recursos hídricos estaduais e de bacias selecionados são planos com horizonte de planejamento de 20 anos, datam de 1998 e portanto ainda não possuem atualizações. Além disso, os três planos de bacias analisados - rio Pontal, Pajeú e Terra Nova - foram também elaborados pela mesma empresa consórcio e têm exatamente os mesmos programas e subprogramas. Esses programas foram elaborados de forma genérica, com poucos detalhamentos sobre recursos, objetivos, órgão e instituições parceiras para execução. E além disso, um horizonte de 20 anos de planejamento parece ser muito longo para as transformações climáticas e dinâmica de uso e gestão das águas nas bacias. O PERH-PE descreve seis componentes, 16 programas e 41 subprogramas. Dos 41 subprogramas, seis foram excluídos da análise, e portanto, 35 foram analisados. A análise indica uma frágil integração entre os planos, porque existe uma baixa interação e sinergia dos subprogramas existentes entre os planos, pois o PERH apresenta apenas um percentual de $31 \%$ de subprogramas iguais aos programas contidos nos PDBHs analisados. 
O Plano Estadual de Alagoas foi elaborado em 2010 e apresenta entre programas e subprogramas, 44 ações. Dentre as quais 24 foram consideradas específicas de âmbito estadual. Apenas o plano do rio afluente Pará foi analisado, donde apresentou também uma frágil coordenação com seu plano estadual. Ou seja, apenas $30 \%$ dos seus programas e/ou subprogramas acataram as diretrizes definidas pelo seu plano estadual de acordo com a Tabela 4.

Tabela 4. Integração por coordenação entre planos estaduais e de bacia de rios estaduais.

\begin{tabular}{llcccccc}
\hline \multirow{2}{*}{ PERH } & \multicolumn{1}{c}{ PDBH } & \multicolumn{3}{c}{ Programas / Subprogramas } & $\begin{array}{c}\text { Programas } \\
\text { iguais (\%) }\end{array}$ & $\begin{array}{c}\text { Coordenação } \\
\text { entre planos }\end{array}$ \\
\hline Minas Gerais & PI & PS & PD & $\begin{array}{c}\text { Total } \\
\text { analisado }\end{array}$ & Score & \\
& Rio Urucuia & 12 & 02 & 00 & 14 & 85,70 & Excelente \\
& Rio Pandeiros & 12 & 02 & 00 & 14 & 85,70 & Excelente \\
& Três Marias & 08 & 05 & 01 & 14 & 57,14 & Boa \\
& Rio das Velhas & 13 & 01 & 00 & 14 & 92,85 & Excelente \\
& Jequitaí e Pacuí & 07 & 04 & 03 & 14 & 50,00 & Boa \\
\multirow{5}{*}{ Pernambuco } & Rio Pará & 07 & 05 & 02 & 14 & 50,00 & Boa \\
& Rio Pontal & 11 & 05 & 19 & 35 & 31,42 & Frágil \\
& Rio Pajeú & 11 & 05 & 19 & 35 & 31,42 & Frágil \\
Alagoas & Rio Terra Nova & 11 & 05 & 19 & 35 & 31,42 & Frágil \\
& Rio Piauí & 06 & 10 & 04 & 20 & 30,00 & Frágil \\
\hline
\end{tabular}

Nenhum plano do estado de Sergipe, Bahia, Goiás e Distrito Federal foram selecionados para análise, pois os mesmos não se enquadravam no critério de seleção. O estado de Goiás e o Distrito Federal não possuem planos diretores de bacias hidrográficas elaborados na região do São Francisco. As áreas destes estados contidas na bacia do rio São Francisco também são relativamente pequenas. A Bahia possui hoje nove planos de bacia a nível estadual (incluindo PERH) e um plano interestadual (BA e MG). Esses planos foram elaborados entre os anos de 1993 e 1996 antes da lei de 1997. O estado na época da elaboração dos planos apresentava uma divisão hidrográfica diferente da atual, os comitês não haviam sido implementados, e, portanto não houve um envolvimento dos atores da bacia na elaboração do plano. Cinco planos estão em fase de elaboração, contratação ou licitação atualmente, a fim de que sob a égide do novo modelo de recursos hídricos estabelecido pela Lei das Águas, implementar um novo cenário de integração entre os planos e articulação entre atores envolvidos.

\subsection{Implementação de Comitês de Bacias}

O segundo aspecto, faz um retrato da implementação dos comitês de bacias de rios estaduais na bacia do rio São Francisco. Existem 44 unidades de planejamento hidrológico de rios afluentes dentro da região do São Francisco (ver Figura 2), 26 não possuem representação de comitês e correspondem a pouco mais de 59\% do número das unidades existentes. Além disso, não existem garantias que todos os comitês implementados são representativos e atuantes, o presente artigo não analisou a real influência e atividades dos comitês nas suas respectivas regiões. Em termo de área, a maior parte da bacia possui comitê de bacia de rio estadual implementado. Já em termos de região, é clara a deficiência no Baixo e Submédio São Francisco, como se pode notar na Figura 2. Essas regiões fisiográficas representam aproximadamente $21 \%$ da área total da bacia e apresentam características particularmente problemáticas, pois estão inseridas no semiárido nordestino com longos períodos de seca; possuem solos com alto risco de salinização devido às atividades agrícolas; apresentam problemas na qualidade da água devido a contaminação fecal e orgânica; importantes hidroelétricas como Paulo Afonso I, II, III e IV, Luiz Gonzaga, Itaparica e Xingó estão 
localizadas nessas regiões, portanto os conflitos entre a geração de energia e outros usos são mais latentes; e os eixos de transposição do rio São Francisco já operam em parte, aumentando a demanda pela água nessa região que apresenta um balanço hídrico superficial crítico (CBHSF, 2016). Portanto, são regiões que enfrentam crescentes conflitos pelo uso da água. Destaque para o estado de Pernambuco que possui 15 bacias de rios estaduais dentro da bacia do São Francisco e apenas uma unidade, a bacia do rio Pajeú, possui comitê instalado.

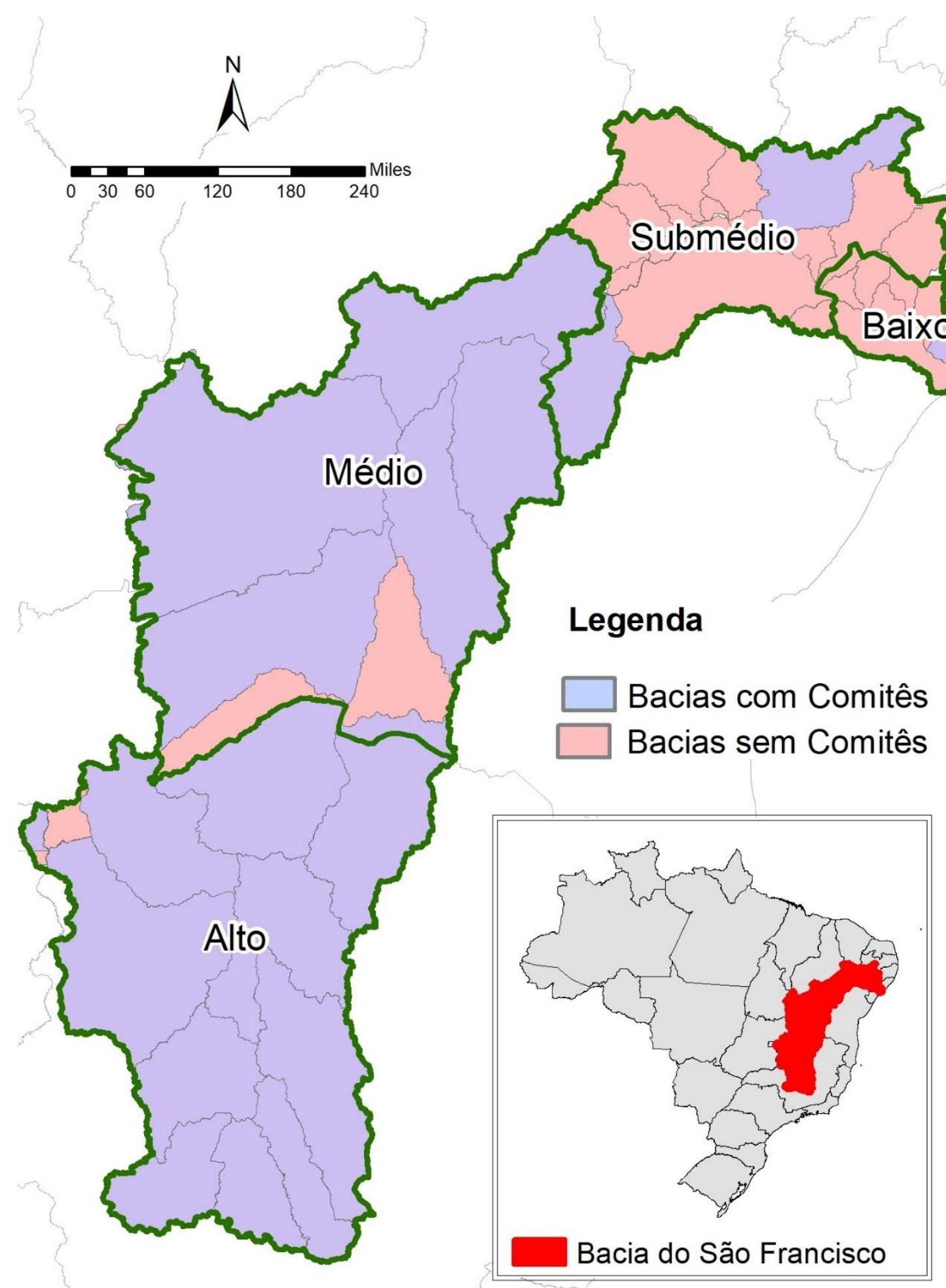

Figura 2. Comitês de bacias implantados no São Francisco e suas novas divisões fisiográficas apresentadas na atualização do PBHSF (CBHSF, 2016). 


\subsection{Acesso e Disponibilidade de Dados nos Web sites}

O terceiro e último aspecto, trata da análise da quantidade, qualidade e autoria das informações. O método é aplicado aos web sites da ANA (Agência Nacional de Águas), INEMA (Instituto de Meio Ambiente e Recursos Hídricos da Bahia), IGAM (Instituto Mineiro de Gestão das Águas), APAC (Agência de Águas e Clima de Pernambuco) e SEMARH-AL (Secretaria de Meio Ambiente e Recursos Hídricos de Alagoas). A Tabela 5 apresenta um quadro da situação baseado nos critérios anteriormente explicados. Nota-se que Bahia, Pernambuco ficam no limite entre uma boa e insuficiente disponibilidade de informações, enquanto que Alagoas apresenta um resultado insuficiente. Os sites desses Estados diferem do sistema de dados da ANA e de Minas Gerais principalmente na exaustividade dos dados, critério no qual expõem a indisponibilidade para acesso direto na plataforma on-line de dados hidrogeológicos, meteorológicos, hidrológicos; sobre os direitos de uso, licenças, outorgas, entre outros.

Tabela 5. Análise das informações disponíveis nos sites institucionais. Adaptado de Codina (2000), Iribarnegaray e Seghezzo (2012) e OECD (2015).

\begin{tabular}{|c|c|c|c|c|c|}
\hline $\begin{array}{l}\text { Qualidade, quantidade e } \\
\text { autoria da informação }\end{array}$ & ANA & $\begin{array}{l}\text { IGAM } \\
\text { MG }\end{array}$ & $\begin{array}{c}\text { INEMA } \\
\text { BA }\end{array}$ & $\begin{array}{l}\text { APAC } \\
\text { PE }\end{array}$ & $\begin{array}{c}\text { SEMAR } \\
\text { AL }\end{array}$ \\
\hline \multicolumn{6}{|c|}{ Nível 1 - Critérios necessários (Peso 1) } \\
\hline 1. Rigor & SIM & SIM & SIM & SIM & NÃO \\
\hline 2. Edição & SIM & NÃO & NÃO & NÃO & NÃO \\
\hline 3. Interesse intrínseco & SIM & SIM & SIM & SIM & SIM \\
\hline 4. Volume & SIM & SIM & SIM & SIM & SIM \\
\hline 5. Responsabilidade & SIM & NÃO & NÃO & NÃO & NÃO \\
\hline \multicolumn{6}{|c|}{ Nível 2 - Critérios notáveis (Peso 2) } \\
\hline 6. Atualização & SIM & SIM & SIM & SIM & SIM \\
\hline 7. Importância & SIM & SIM & SIM & SIM & SIM \\
\hline \multicolumn{6}{|c|}{ Nível 3 - Critérios excelentes (Peso 3) } \\
\hline 8. Exaustividade & SIM & SIM & $\mathrm{NA} \mathrm{O}$ & NÃO & NÃO \\
\hline 9. Sistematização & NÃO & NÃO & NÃO & NÃO & $\mathrm{NAO}$ \\
\hline 10. Originalidade & SIM & SIM & SIM & SIM & SIM \\
\hline Score & $83,33 \%$ & $72,22 \%$ & $55,55 \%$ & $55,22 \%$ & $50,00 \%$ \\
\hline $\begin{array}{l}\text { Acesso e disponibilidade de } \\
\text { dados de web sites (WS) }\end{array}$ & Excelente & Boa & Boa & Boa & Insuficiente \\
\hline
\end{tabular}

\section{CONCLUSÃO}

A bacia do rio São Francisco apresenta fragilidades no quesito governança multinível. Algumas regiões ainda precisam conciliar seus limites administrativos e hidrológicos, como por exemplo, definir claramente regras e responsabilidades em rios donde dois ou mais órgãos de gestão de recursos hídricos são responsáveis por trechos diferentes. Essa falta de clareza gera incompatibilidades gerenciais, fragmentação institucional e uma abordagem hídrica em escala inadequada. Consequentemente, os instrumentos de gestão estabelecidos pela política nacional ainda enfrentam diversos entraves para sua efetiva implementação, passados 20 anos da aprovação da lei das águas. Em escala estadual - principalmente nas regiões do Submédio e Baixo São Francisco - destacam-se os desafios na articulação vertical entre os planos estaduais e planos de bacias dos rios afluentes. Falta integração entre os planos em suas diferentes escalas; ora pela falta de diálogo entre conselho, comitês de bacias, órgãos gestores e agências de bacias; 
ora pela lacuna na participação pública e dos usuários de água. A maioria dos planos dos rios afluentes vigentes foi elaborado pelo poder público. Consequentemente, uma boa parte dos planos analisados apresentam propostas com divergências regionais, apresentam falhas na sinergia das ações e programas concorrentes nos diferentes níveis de planejamento. Faz-se também necessário aprimorar o mecanismo de articulação entre os planos horizontais, como por exemplo, a articulação entre os planos diretores municipais e planos de bacias. Outro aspecto importante é a eficácia dos planos, não bastaria uma boa articulação entre níveis e instituições sem que os planos não suprissem as necessidades da bacia, ou que seus programas não fossem viáveis de implementação. É necessário avaliar a qualidade e horizonte de planejamento dos planos, muitos deles chegam a ser elaborados para 20 anos e ficam notadamente incompatíveis com a dinâmica apresentada pelas bacias e apresentam ações genéricas de difícil implementação. Para que os planos estaduais (PERHs) de fato representem toda a diversidade de suas bacias de rios afluentes, há de se observar, dentre outros, a dimensão territorial do estado, sua localização na divisão hidrográfica interestadual, a localização dos seus usuários, o impacto de usos da água e o número de comitês instalados e atuantes nas subbacias.

Ainda com destaque para o Submédio e Baixo, muito dos comitês não foram sequer implementados ou não foram instalados em harmonia com a elaboração dos seus planos de bacias, por exemplo, nos estados da Bahia, Sergipe, Alagoas e Pernambuco os planos foram elaborados pelos órgãos estaduais e muitos deles no momento da preparação não existiam comitês instalados. Portanto, parte da região do São Francisco apresenta uma lacuna na participação dos atores da bacia na definição das metas para a racionalização de uso e na construção de diretrizes gerais que atendam os instrumentos definidos na Política Nacional de Recursos Hídricos. No que se diz respeito à disponibilização de informações relativas à gestão de água, no geral os sites analisados apresentaram uma boa plataforma de disponibilização de dados, porém as instituições governamentais analisadas no Submédio e Baixo São Francisco ficam no limite entre uma boa e uma insuficiente disponibilização de dados. Diante disso, fica claro que os aspectos aqui avaliados foram representativos e demonstraram que a problemática da governança hídrica é mais latente nas regiões do semiárido, pois é onde apresentam os piores desempenhos. E é exatamente, não por coincidência, onde as disparidades sociais e os conflitos pelo uso da água são mais proeminentes.

Os aspectos abordados foram suficientes para embasar uma análise comparativa entre as regiões dentro da bacia, fazendo uma constatação para a real possibilidade de se construir um indicador, considerando aprimoramentos, para mensurar o desempenho da governança nas diferentes regiões do São Francisco. Por outro lado, para se mensurar a boa governança, o artigo carece de uma maior completude dos seus tópicos avaliados, como por exemplo, aferir a falta de compatibilização entre fronteiras hidrológicas e administrativas; mensurar a fragmentação setorial das tarefas relacionadas com a gestão da água; estimar a lacuna de capacitação, principalmente em nível local donde os técnicos necessitam de maior investimento em treinamento; medir a problemática da origem de recursos e dos investimentos instáveis; entre outros. Vale ainda ressaltar, que mesmo que haja a garantia da elaboração de planos coordenados, com a devida participação dos atores da bacia e um horizonte de planejamento coerente, ainda sim sua eficiência depende de sua correta implementação, que envolva fatores como alocação de recursos financeiros, prazo, priorização e monitoramento para a execução das ações, entre outros aspectos.

Recomenda-se o uso de ferramentas que possa melhorar a articulação entre os planos de gestão hídrica e gestão territorial, dessa forma pode se auxiliar na melhoria da gestão compartilhada entre diferentes escalas. A aplicação da Avaliação Ambiental Estratégica durante a preparação dos planos de bacias seria uma metodologia indicada, uma vez que a ferramenta considera todas as políticas, planos e programas setoriais que interferem e se relaciona com os 
planos de bacias. Outra recomendação é em relação ao grau de envolvimento dos atores institucionais e individuais em espaços de interação, onde o envolvimento do público com as autoridades de água torna-se possível. Esse ponto toca na disponibilidade de informações para os usuários e partes interessadas da bacia e no fortalecimento dos espaços para debates e tomadas de decisão. Apesar de boa parte das instituições apresentarem plataformas online com dados relevantes, é necessário uma plataforma de compartilhamento de dados colaborativa, onde diferentes setores da sociedade possam contribuir. E com isso, dar uma maior visibilidade e acessibilidade às informações geradas na bacia, como por exemplo, pelos pesquisadores nas universidades, nas ONGs, nas cooperativas, associações, comitês, entre outros. A padronização dessas plataformas de acesso e dessas informações, que poderiam ser gerenciadas pelos orgãos estaduais de gestão de água, também é um ponto relevante nesse processo. Outro espaço de compartilhamento de informações - os comitês - continua sendo a instancia mais relevante para discussões, principalmente em escala local de bacias dos rios afluentes. Recomenda-se o afinco dos estados em fortalecer esse instrumento, não só oficializando a implementação, mas também mantendo o colegiado atuante.

Por fim, as dimensões da bacia do São Francisco traz disparidades econômicas, sociais, culturais e climáticas. Essa heterogeneidade tem dificultado a gestão compartilhada e descentralizada, bem como as necessidades específicas das sub-bacias não interagem de forma relevante com o curso do rio principal. Adicionalmente, a crise hídrica na bacia não é só de falta d'água e sim de práticas insustentáveis no uso desse recurso e de conflitos de interesse entre usuários. Para superar essas dificuldades faz-se necessário investir não apenas nesses aspectos abordados no presente artigo, mas também em outros indispensáveis princípios da governança, como por exemplo, na capacitação dos usuários, na questão do papel das mulheres na tomada de decisão, na clareza e na distinção das responsabilidades na formulação de política pública e na sustentabilidade dos recursos financeiros da bacia, dentre outros.

\section{AGRADECIMENTOS}

Ao Projeto INNOVATE (BMBF, n01LL0904A), CNPq (248569/2013-9). Pelo apoio intelectual, disponibilização de dados, espaço cedido para pesquisa e aporte financeiro.

\section{REFERÊNCIAS}

AGÊNCIA NACIONAL DE ÁGUAS - ANA (Brasil). Planos de recursos hídricos e enquadramento dos corpos de água: caderno de capacitação em recursos hídricos. Brasília, 2013. 68 p.

BISWAS, A. K.; TORTAJADA, C. Future water governance: problems and perspectives. International Journal of Water Resources Development, v. 26, n. 2, p. 129-139, 2010. http://dx.doi.org/10.1080/07900627.2010.488853

CARTER, N.; KREUTZWISER, D. R.; LOË, R. C. Closing the circle: linking land use planning and water management at the local level. Land Use Policy, v. 22, p. 115-127, 2004. https://doi.org/10.1016/j.landusepol.2004.01.004

CODINA, L. Evaluación de recursos digitales em línea: conceptos, indicadores y métodos. Revista Española de Documentación Científica, v. 1, p. 23-58, 2000.

COMITÊ DA BACIA HIDROGRÁFICA DO RIO SÃO FRANCISCO - CBHSF. Plano de recursos hídricos da bacia hidrográfica do São Francisco: resumo executivo do plano de recursos hídricos. 2016. Disponível em: http://cbhsaofrancisco.org.br/planoderecursoshidricos/relatorios/. Acesso em: 02 outubro 2016. 
IRIBARNEGARAY, A. M.; SEGHEZZO, L. Governance, sustainability and decision making in water and sanitation management systems. Sustainability Journal, v. 4, p. 2922-2945, 2012. http://dx.doi.org/10.3390/su4112922

LANNA, A. E. L.; PEREIRA, J. S.; HUBERT, G. Os novos instrumentos de planejamento do sistema francês de gestão de recursos hídricos: II - Reflexões e propostas para o Brasil. Revista Brasileira de Recursos Hídricos, v. 7, n. 2, p. 109-120, 2002. http://dx.doi.org/10.21168/rbrh.v7n2.p109-120

MITCHELL, B. Integrated water resource management, institutional arrangements, and landuse planning. Environment and Planning A, v. 37, p. 1335-1352, 2004. https://doi.org/10.1068\%2Fa37224

MOSS, T. The governance of land use in river basins: prospects for overcoming problems of institutional interplay with the EU Water Framework Directive. Land Use Policy, v. 21, p. 85-94, 2003. http://dx.doi:10.1016/j.landusepol.2003.10.001

NESHEIM, I.; MCNEILL, D.; JOY, K. J.; MANASI, S.; NHUNG, D. T. K.; PORTELA, M. M. et al. The challenge and status of IWRM in four river basins in Europe and Asia. Irrigation Drainage Systems, v. 24, p. 205-221, 2010. https://doi.org/10.1007/s10795010-9103-9

NEVES, M. J.; CORDEIRO NETTO, O. M. Avaliação de efetividade de planos de recursos hídricos desenvolvidos no Brasil. In: SIMPÓSIO DE RECURSOS HÍDRICOS DO NORDESTE, 16, 2005, João Pessoa. Porto Alegre: ABRH, 2005.

ORGANIZAÇÃO PARA A COOPERAÇÃO E DESENVOLVIMENTO ECONÓMICO OECD. Principles on water governance. 2015. Disponível em: http://www.oecd.org/gov/regional-policy/OECD-Principles-on-Water-Governancebrochure.pdf. Acesso em: 15 setembro 2015.

SIEGMUND-SCHULTZE, M.; RODORFF, V.; KÖPPEL, J.; SOBRAL, M. C. Paternalism or participatory governance? Efforts and obstacles in implementing the Brazilian water policy in a large watershed. Land Use Policy, v. 48, p. 120-130, 2015. http://dx.doi.10.1016/j.landusepol.2015.05.024.

SILVA, S. R.; CIRILO, A. C. O planejamento de recursos hídricos na bacia do rio são Francisco. Revista de Gestão de Água da América Latina - REGA, v. 8, n. 1, p. 47 64, 2011.

VEIGA, L. B. E.; MAGRINI, A. The Brazilian water resources management policy: Fifteen years of success and challenges. Water Resources Management, v. 27, p. 2287-2302, 2013. http://dx.doi.1007/s11269-013-0288-1 